\title{
Time-till-breakdown and scalp electrical potential maps of long-range apparent motion
}

\author{
C. M. SELMES, W. R. FULHAM, D. C. FINLAY, and M. C. CHORLTON \\ University of Newcastle, Newcastle, New South Wales, Australia \\ and \\ M. L. MANNING \\ Griffith University, Southport, Queensland, Australia
}

\begin{abstract}
A series of psychophysical and electrophysiological experiments is reported using the apparent motion (AM) breakdown effect. Breakdown describes an effect in AM in which, during continuous viewing, the percept of smooth motion of a single stimulus alternates with the percept of two discrete alternating stimuli. Visual evoked potentials (VEPs) were recorded during periods of motion or breakdown ("nonmotion") in horizontal and vertical displays. VEPs were compared with synthetic VEPs ("composite-flash") produced by adding VEPs to each element of the display recorded in isolation. Subtraction of VEPs was used in an attempt to compare the electrical responses with the processing of information relating to the form of the stimulus, subthreshold motion processing, and suprathreshold motion processing. The results, presented as scalp electrical potential distribution maps, were interpreted as consistent with a central adaptation process underlying the breakdown effect. The results also indicated that the hemispheric asymmetries in AM VEPs described by Manning, Finlay, and Fenelon (1988) were most likely due to the position of the stimuli in the visual field, rather than as a lateralization of motion processes per se. The results also provided evidence that the subthreshold and suprathreshold motion responses to the display were the product of different populations of motion units.
\end{abstract}

Braddick (1974) proposed a dichotomy in apparent motion (AM) processes-namely, a long-range apparent motion (LRAM) process that was attributed to a higher order interpretive mechanism, and a far more specific short-range apparent motion (SRAM) process that was attributed to a passive low-level mechanism. The distinction remains controversial, however, with some authors disputing its usefulness and instead arguing that SRAM, LRAM, and real motion (RM) are all processed by the same neural mechanism(s). Burr and colleagues (Burr \& Ross, 1986; Burr, Ross, \& Morrone, 1986), for example, have proposed that receptive field processes of varying visual field width constitute the underlying mechanism, although Manning and Mazzucchelli (1992) have recently presented evidence suggesting that a dichotomy between SRAM and LRAM cannot be explained in simple terms of spatial parameters.

Kolers (1964) observed that during prolonged viewing of simple two-element AM displays, the motion percept was subject to periods of breakdown, or reversion to the percept of isolated flashing of the stimulus components. Frisby (1972) argued that this phenomenon is the result of motion processors operating at near-tolerance levels during stimulation by $\mathrm{AM}$, thereby rendering a percept

This research was supported by ARC Grant 42/280/025. Correspondence should be addressed to D. C. Finlay, Department of Psychology, University of Newcastle, NSW, Australia, 2308 (e-mail: finlay@psychology.newcastle.edu.au). susceptible to the effects of adaptation with the output of these units falling below the level required for the maintained perception of movement. Frisby also drew support for this interpretation from reports that motion perception is more common during the initial stages of viewing than it is during the later stages, a point that he suggested was reminiscent of the fatigue effects found by Barlow and Hill (1963) in motion-sensitive neurons in the rabbit retina. The latter point is perhaps more aptly reflected in Vautin and Berkley's (1977) subsequent finding of a progressive reduction in neuronal responsiveness in cat striate cortex following prolonged exposure to a moving stimulus.

Clatworthy and Frisby (1973) suggested that highlevel motion processes are responsible for breakdown, since adaptation to RM or AM renders similar effects on subsequent AM viewing, whereas adaptation to in -phase flash or continuously illuminated stimuli produces a much reduced effect. Support for an association between AM breakdown and a centrally located mechanism has also been derived from demonstrations that stereoscopically fused stimuli in motion exhibit breakdown (Finlay, Manning, Neill, \& Fenelon, 1987), and also from observations that AM produces a motion aftereffect, also the result of adaptation, under dichoptic viewing conditions (von Grünau, 1986).

Animal models have also provided evidence of a centrally located mechanism (Maffei, Fiorentini, \& Bisti, 1973; Vautin \& Berkley, 1977). Moreover, Mikami (1991) proposed that the motion system is likely to be hierar- 
chically organized, suggesting that cortical regions V1 and perhaps $V 2$ constitute the underpinnings of the SRAM process. Mikami proposed that the LRAM process, on the other hand, is associated with an area analogous to the middle temporal area (MT) of the macaque, an area that has provided the strongest evidence to date of localized specialization within the visual system because of its regional domination by direction selective neurons (Wurtz, Yamasaki, Duffy, \& Roy, 1990).

Psychophysical investigations have used the breakdown effect as a means of deriving the "strength" of the AM percept following manipulation of various stimulus parameters. Different indices of breakdown, however, have been employed in different studies. Anstis, Giaschi, and Cogan (1985), for example, employed the probability of seeing motion over fixed time periods, whereas Finlay and von Grünau (1987) used a time-till-breakdown (TTB) measure of AM strength, or the length of time motion is perceived before breakdown occurs for the first time. Although the two studies used different indices, they produced consistent psychophysical functions of AM strength across different temporal frequencies. Using TTB, Finlay and von Grünau found that LRAM was perceived within a limited temporal frequency range, or "envelope," of approximately 1.0 to $5.0 \mathrm{~Hz}$, with the motion percept being most resistant to breakdown between 2.0 and $3.0 \mathrm{~Hz}$ and particularly vulnerable toward the flanks of the envelope, especially at the upper limits. Moreover, these temporal parameters remained unchanged irrespective of manipulation to stimulus duration and interstimulus interval (ISI)that is, when stimulus duration and ISI were varied, but frequency, and hence stimulus onset asynchrony (SOA; stimulus duration + ISI), was kept constant, TTB also remained constant.

Pantle (1974) came to a similar conclusion after finding an interaction between pattern characteristics and adapting speed and suggested that the responses of mechanisms sensitive to direction in humans were under the control of the temporal frequency of a drifting pattern rather than its velocity. Finlay and von Grünau (1987) also proposed that the breakdown effect was due to adaptation, or neuronal fatigue, since continuous stimulation led to faster breakdown.

Steinman and Levi (1992) noted that many studies of spatial vision have confounded interpretation of visual evoked potential (VEP) data through the use of vastly different stimulus arrangements. Studies attempting to investigate AM phenomena have used different stimulus arrangements to compare motion with nonmotion VEP responses (Andreassi, Mayzner, Stern, and Okamura, 1973; Coffin, 1977). Using a continuously cycling (3Hz) LRAM display, however, Manning, Finlay, and Fenelon (1988) recorded VEPs while motion was reported and, with the identical stimulus situation, during periods of breakdown, or "nonmotion," thereby avoiding this problem.

Waveforms from six scalp sites $(\mathrm{O} 1, \mathrm{O} 2, \mathrm{~T} 5, \mathrm{~T} 6, \mathrm{P} 3$, and P4) were found to be similar for both the motion and breakdown conditions. Two motion-sensitive compo- nents were identified. The first was a positive-going component at $60-82 \mathrm{msec}$ post left-field stimulus, and the second was a negative-going component 100-126 msec post right-field stimulus. In both cases the motion components were of greater amplitude than were the nonmotion. Significant differences between motion and nonmotion were found for right-hemisphere but not lefthemisphere sites. As component amplitudes were greater in periods in which the subjective percept was of motion, rather than nonmotion, we interpreted the results as supporting the notion that adaptation is the underlying basis of the breakdown effect.

We proposed that the results were, in general, consistent with animal studies of RM single unit responses in which the initial extraction of information relating to LRAM takes place within the occipital lobe, with further processing within temporal and parietal lobes. Activity in temporal and parietal lobes was, however, largely lateralized to the right hemisphere. Only limited evidence has been reported from psychophysical studies suggesting asymmetries in AM perception. Eals (1987) reported that subjects were more likely to predict the correct continuation of an AM trajectory when stimuli were presented in the left hemifield than in the right. Eals concluded that there is a right cortical dominance for AM processing.

The present study aims to use the AM breakdown effect to gather motion and nonmotion VEPs elicited by both horizontally and vertically oriented simple twoelement AM displays. It is hoped that the comparison of VEPs elicited by horizontal and vertical stimulus arrays will help resolve whether the asymmetries in VEP responses reported by Manning et al. (1988) were the result of a lateralization of motion processing per se or in some way reflected responses to that particular horizontal display.

This study also compares "motion VEPs," gathered during periods when subjects perceive motion from the display, and "nonmotion VEPs," gathered during periods of breakdown with synthetic nonmotion VEPs. These synthetic, or "composite-flash" VEPs, were produced by collecting VEPs in response to a single element of a twoelement AM display (e.g., a "right" element from the horizontal display) and adding these VEPs to VEPs recorded in response to the corresponding second element of a two-element display (the "left" element in this example). These composite-flash VEPs were produced in an attempt to provide a comparison VEP that would contain the responses relevant to the processing of form information from the display without any accompanying suprathreshold or subthreshold motion related electrical activity that would be expected to be present in motion and nonmotion VEPs.

The assumptions that underlie this approach obviously involve the linearities of the system and the VEPs generated, and fortunately relevant data are available. In order to examine the recovery function of the "flash" VEP, Schwartz and Shagass (1964) recorded responses to conditioning and test stimuli at graded ISIs and sub- 
sequently linearly subtracted responses to the conditioning stimulus from those recorded to the paired stimuli. An examination of VEP waveform, amplitude, and latency indicated that the first two measures, waveform and amplitude, show recovery at approximately $100 \mathrm{msec}$, whereas latency requires in excess of $120 \mathrm{msec}$ for recovery - periods that are shorter than ISIs to be used in the electrophysiological experiments here.

For both horizontal and vertical displays, three sets of VEPs were produced. First, composite-flash VEPs were interpreted, for the purpose of comparison, as representing the responses to the onset and offset of form characteristics of the stimuli. Second, the nonmotion VEPs gathered during periods of breakdown for the AM displays were interpreted as representing the combined response of (1) the onset and offset of form characteristics of the stimuli and (2) the subthreshold responses of cortical motion processing. Third, the VEPs gathered during periods of motion for the AM displays were interpreted as representing the combined responses of (1) the onset and offset of form characteristics of the stimuli, (2) the subthreshold responses of cortical motion processing, and (3) the suprathreshold responses of cortical motion processing to the LRAM display.

In an attempt to examine the distribution of form, subthreshold motion processing, and suprathreshold motion processing of the AM displays, difference VEPs were calculated by subtractions of these three classes of VEP.

\section{EXPERIMENT 1}

The purpose of Experiment 1 was to determine, for the stimuli used here, the variation in TTB as a function of temporal frequency, since Finlay and von Grünau (1987) showed temporal frequency or SOA to be a more important determinant of TTB than stimulus duration or ISI. Additionally, Experiment 1 also served to familiarize those subjects participating in Experiments 2 and 3 with the perception of LRAM.

\section{Method}

Subjects. Thirty-eight subjects between 19 and 37 years of age participated. All were right-handed and had normal or correctedto-normal vision.

Stimuli and Apparatus. An Amiga 1000 computer and 1084S monitor were used for stimulus presentation and response recording. Subjects made responses using a three-button control box.

The stimulus display consisted of two vertical white rectangles, each subtending $1.37^{\circ} \times .54^{\circ}$, with center-to-center separation of $2.1^{\circ}$. The stimulus luminance was $8.8 \mathrm{~cd} / \mathrm{m}^{2}$ against a black background. Ten temporal frequencies were used: 1.0, 1.5, 2.0, 2.5, 2.7, $3.1,3.7,4.2,5.0$, and $6.25 \mathrm{~Hz}$. The on-time for each rectangle remained constant at $60 \mathrm{msec}$, and ISIs ranged from $20 \mathrm{msec}$ for $6.25 \mathrm{~Hz}$ up to $440 \mathrm{msec}$ for the $1.0-\mathrm{Hz}$ condition. A central fixation point appeared on the screen throughout the experimental session.

Procedure. All testing was carried out individually under dimmed lighting conditions at a viewing distance of $2.0 \mathrm{~m}$. Subjects were instructed to fixate, initiate a trial when ready, and to use the different response keys to indicate the onset of LRAM perception and, if necessary, the onset of breakdown. In the latter case, the trial was immediately discontinued, whereas in the absence of LRAM or breakdown, each trial ceased automatically after $60 \mathrm{sec}$. Subjects were given a series of 10 frequency-randomized practice trials, which were followed by 100 randomized test trials comprising 10 trials of each frequency. Subjects participating in Experiment 2 attended this experimental session 3 to 4 days prior to the subsequent study.

\section{Results}

Mean TTB - that is, the time from the report of motion until breakdown or, where breakdown does not occur, until the end of the 60-sec trial-was calculated for each temporal frequency across all subjects and is shown in Figure 1. A stimulus frequency of $2.7 \mathrm{~Hz}$ resulted in the longest TTB. A one-way repeated measures analysis of variance (ANOVA; BMDP2V-Dixon, 1985) conducted on mean TTB data revealed a significant effect $[F(9,333)=9.22, p<.01]$ for stimulus frequency. Post hoc analysis (Tukey's HSD) demonstrated that frequencies of $2.0,2.5,2.7$, and $3.1 \mathrm{~Hz}$ differed significantly from $1.0,4.2,5.0$, and $6.2 \mathrm{~Hz}$.

The results of Experiment 1 indicated that a temporal frequency of $2.7 \mathrm{~Hz}$ led to the longest periods of motion perception and so may be described as rendering the greatest LRAM strength or stability. Furthermore, post hoc analyses indicated a temporal envelope existing from 2.0 to $3.1 \mathrm{~Hz}$ where the LRAM percept is particularly resilient.

\section{EXPERIMENTS 2 AND 3}

Although Experiments 2 and 3 were conducted separately, they are reported in conjunction because their experimental and analytical procedures were similar. Experiment 2 investigated the effect of motion perception on VEPs to a horizontally oscillating LRAM display, and Experiment 3 investigated a vertically oscillating LRAM display.

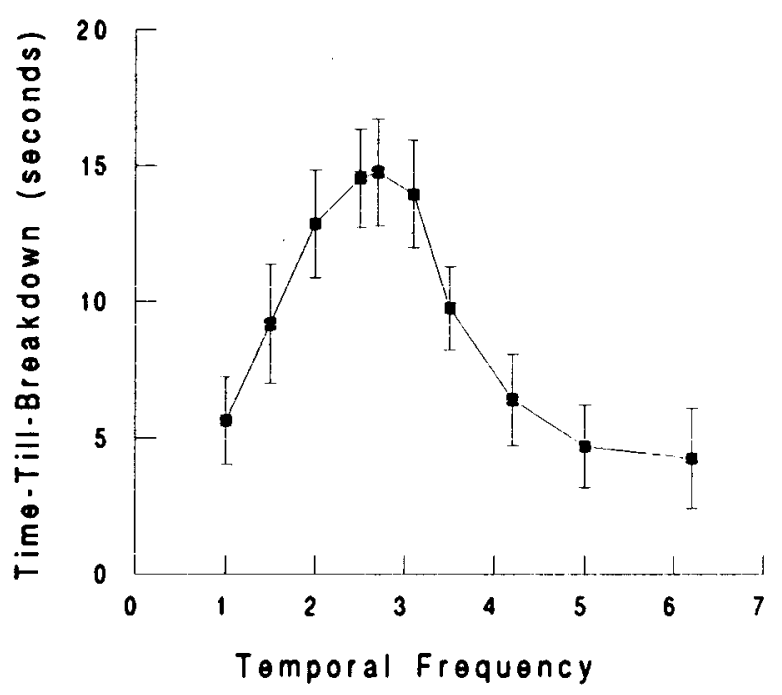

Figure 1. Mean time-till-breakdown as a function of temporal frequency (vertical bars indicate $S E M$ ). 


\section{Method}

Subjects. Fourteen subjects ( 7 male and 7 female) between the ages of 20 and 35 years participated in Experiment 2, the horizontal motion condition, and 6 of these same subjects ( 4 male and 2 female), between 21 and 36 years, also participated in Experiment 3 , the vertical motion condition. All were right-handed and had normal or corrected-to-normal vision. These subjects had also participated in Experiment 1, and since all subjects taking part in the initial experiment experienced reasonably lengthy periods of LRAM perception, recruitment for the latter studies was based solely on the subject's availability.

Apparatus and Stimuli. A Commodore 500 microcomputer was interfaced with an IBM-compatible microcomputer that recorded VEP data; together these were used to control the presentation of experimental blocks. An Amiga 1084S monitor was used for stimulus display.

Stimuli in Experiment 2 (horizontal LRAM) were identical to those used in Experiment 1. A single frequency of $2.5 \mathrm{~Hz}$ was employed, with stimulus on-time of $60 \mathrm{msec}$ and an ISI of $140 \mathrm{msec}$ being used in the LRAM condition. Two additional conditions were included in Experiment 2, in which stimulus displays consisted of a single rectangle of the same dimensions as those used in the LRAM condition, with center offset $1.05^{\circ}$ either to the left or right of the fixation point (left-flash and right-flash conditions, respectively). The flash frequency was set to match that of the left (or right) stimulus element in the AM display. For the left-flash condition, for example, a left stimulus on-time of $60 \mathrm{msec}$ was followed by an ISI of $340 \mathrm{msec}$.

The same stimulus arrangements as those used in Experiment 2 were used in Experiment 3 except that the display was physically rotated through $90^{\circ}$ so that the rectangles were horizontally oriented and located above and below the fixation point. This produced three stimulus arrangements-vertical LRAM, upper flash, and lower flash. A single push button was provided for the subject's responses to the horizontal LRAM and vertical LRAM displays.

Electrophysiological technique. Sixteen gold electrodes were attached to the scalp with collodion and filled with conductive gel. The montage (Figure 2) used standard international 10-20 electrode system sites $\mathrm{Oz}, \mathrm{O} 1, \mathrm{O} 2, \mathrm{Pz}, \mathrm{P} 3, \mathrm{P} 4, \mathrm{~T} 5$, and $\mathrm{T} 6$, together with placements at $\mathrm{Oz}+$ (midway between $\mathrm{Oz}$ and $\mathrm{Pz}), \mathrm{O} 1-(10 \%$ below $\mathrm{O} 1), \mathrm{O} 2-(10 \%$ below O2), O1.T5- (20\% along the left inion-nasion circumference), O2.T6- (20\% along the right inionnasion circumference), IP3 (two thirds of the distance from P3 to T3), and IP4 (two thirds of the distance from P4 to T4), right and left mastoids; an earth electrode was positioned on the forehead. The left mastoid was used as the reference electrode.

Electrical impedances were reduced to below $5 \mathrm{k} \Omega$, and filters set at $0.3-$ to $100-\mathrm{Hz}$ bandpass. Electrophysiological signals had a sampling rate of $2 \mathrm{msec}$ and were amplified $(\times 50,000)$ and stored as single trials of 1.6-sec duration ( 30 of these trials constituted one block). A Grass Model 12, 16-channel amplifier, interfaced

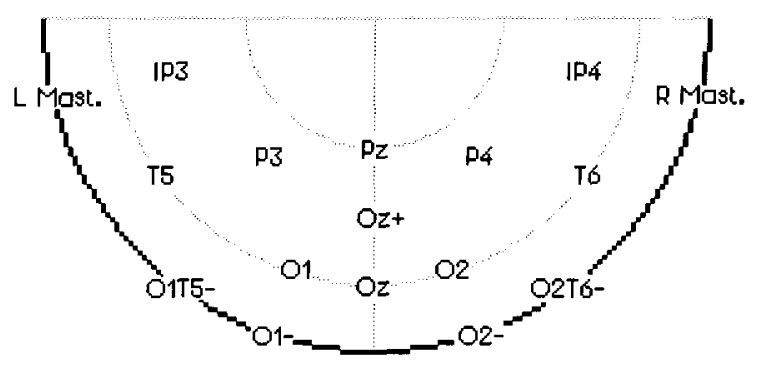

Figure 2. Electrode positions presented on the same projection used to present scalp electrical potential distribution maps. Mast., mastoid. (via a Data Translation DT-2801/A analog to digital card) to an IBM-compatible microcomputer running ASYST software, recorded VEP data.

Procedure. For the LRAM condition, subjects were instructed to fixate and to depress a right-hand-held response key during the percept of LRAM and to release it during the percept of breakdown. The computer coded successive 1.6-sec EEG records as being either motion or nonmotion-or, alternatively, as a trial in which a button transition occurred. In the latter case, the trial was rejected on line, as were any trials in which large eye movements occurred. Electrophysiological data sampling was not synchronized to buttonpress or release. During left-right and upper-lower flash conditions, no behavioral response was required; subjects merely maintained fixation throughout the trial.

The experimental session was composed of a randomized presentation of seven blocks of either horizontal or vertical LRAM, together with three blocks each of the left- and right-flash (Experiment 2), and upper- and lower-flash (Experiment 3) conditions (one practice block of the LRAM condition was provided at the commencement of each session). A rest period of approximately $3 \mathrm{~min}$ was provided between blocks, and each session required around $1.25 \mathrm{~h}$ to complete.

\section{Results}

Analysis of adaptation effects. Overall, both horizontal and vertical LRAM conditions indicated similar ratios of motion to nonmotion in experimental blocks (i.e., for the horizontal LRAM condition, motion was perceived during $60 \%$ of trials, and $40 \%$ were coded as nonmotion trials, whereas for the vertical LRAM condition, $56 \%$ were coded as motion trials and $44 \%$ as nonmotion trials).

It is being assumed here that any decrease in the proportion of motion being perceived over time may be a behavioral measure or reflection of adaptation of motion processors. Therefore, in order to place constraints on the analysis of VEPs across subjects, a procedure similar to that followed by Manning et al. (1988) was used to control for extraneous fatigue effects and differing signal-to-noise ratios resulting from subjects' experiencing differing amounts of motion and nonmotion during the course of the block. That is, for each subject, the seven LRAM blocks were each partitioned into six 8-sec divisions and an equal number of motion-nonmotion and flash trials were selected from each division. Subjects' individual data were then combined and grand averages were calculated for each stimulus condition.

Examination of the mean proportion of motion perceived throughout the experimental session failed to reveal any effect due to the position of each block within the session, suggesting that fatigue and adaptation need not be considered at this level. In relation to temporal divisions within these blocks, however, chi-square analyses carried out on the number of trials coded either as motion or nonmotion in each of the six divisions of the matched data revealed a significant effect in both the horizontal $\left[\chi^{2}(5, N=2,197)=59.00, p<.05\right]$ and vertical $\left[\chi^{2}(5, N=1,047)=34.96, p<.05\right]$ LRAM conditions. The mean proportion of trials in which motion was reported is displayed in Figure 3. The pattern of results for the two conditions was highly correlated $(r=.81, p<$ .05 ), and Figure 3 demonstrates that, apart from the first 


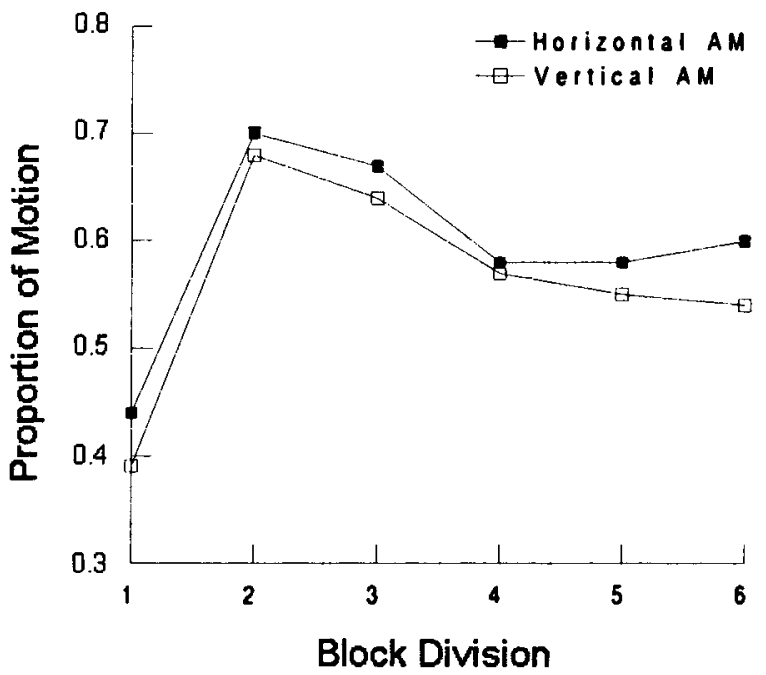

Figure 3. Mean proportion of motion perceived in each of the six temporal divisions imposed on the horizontal and vertical apparent motion (AM) blocks (each block division represents an 8-sec recording period).

division, progressively less motion was perceived as the experimental block proceeded for both horizontal and vertical LRAM conditions, suggesting that adaptation may be having an effect at this level.

Comparison of horizontal LRAM VEP motion components with those of Manning et al. (1988). VEP responses containing artifact were rejected prior to data analysis and waveforms rereferenced off line to averaged mastoids. Average VEPs were calculated for each condition for each subject. Grand average VEPs were calculated across subjects for each stimulus condition, rendering a 400-msec, one-stimulus cycle waveform, with left or upper stimulus onset at time zero. Since this is a steady-state VEP whose mean amplitude is conventionally treated as zero, baseline correction was performed by subtracting the average value across one full stimulus cycle.

In the experiment conducted here, we used stimuli alternating at a slightly different temporal frequency $(2.5 \mathrm{~Hz})$ and so the VEPs presented here are not directly comparable with those of Manning et al. (3 Hz; 1988), who found two motion-sensitive components for their horizontal display. The first was a positive-going component at $60-82 \mathrm{msec}$ post left-field stimulus, and the second was a negative-going component $100-126 \mathrm{msec}$ post right-field stimulus. Visual inspection of our data (Figure 4), however, revealed differences between motion and nonmotion VEPs at similar regions in our waveforms: a positive-going region at around $50-70 \mathrm{msec}$ post left-field stimulus (50-70 msec into the recording epoch) and a negative-going region at around $90-110 \mathrm{msec}$ post right-field stimulus $(290-310 \mathrm{msec}$ into the recording epoch). Figure 4a presents motion and nonmotion VEPs in response to the horizontal motion display for the same six scalp sites reported by Manning et al. (1988).

For the purpose of comparison with previously reported results, statistical comparisons were made be- tween motion and nonmotion responses from the six scalp sites for which results were previously reported by Manning et al. (1988). For the six scalp sites (O1, O2, $\mathrm{T} 5, \mathrm{~T} 6, \mathrm{P} 3$, and $\mathrm{P} 4)$ at each of these windows (50-70 and 290-310 msec), mean values were found for each subject, and $2 \times 2 \times 3$ (motion $\times$ hemisphere $\times$ lobe) repeated measures ANOVAs (BMDP2V-Dixon, 1985) were conducted for each window.

1. The 50- to 70-msec window: A significant main effect was found for lobe $[F(2,26)=6.81, p=.0042$; occipital $=-.388$, temporal $=-.0651$, parietal $=0.042]$. The motion $\times$ hemisphere interaction effect was also significant $[F(1,13)=12.27, p=.0039]$.

Given the results of Manning et al. (1988), we had an a priori expectation as to the direction of differences in amplitude between motion and nonmotion VEPs, and so post hoc comparisons on the interaction effect were conducted using one-tailed $t$ tests. In the right hemisphere, motion responses $(M=0.166 \mu \mathrm{V})$ of the positive-going component were of a significantly larger amplitude than were nonmotion responses $(M=-0.337 \mu \mathrm{V})[t(13)=$ $2.09, p<.03$ ]. In the left hemisphere, however, there was no significant difference between motion $(M=$ $-0.251 \mu \mathrm{V})$ and nonmotion $(M=-0.126 \mu \mathrm{V})$ amplitudes $[t(13)=-0.58, p>.05]$.

2. The 290- to 310-msec window: A significant main effect was found for motion $[F(1,13)=5.55, p=.0348]$, with motion responses of the negative-going component of greater amplitude $(M=-0.074 \mu \mathrm{V})$ than nonmotion responses $(M=1.101 \mu \mathrm{V})$. The motion $\times$ lobe interaction effect was also significant $[F(2,26)=4.52, p=$ .0207].

Post hoc comparisons on the interaction effect were conducted using one-tailed $t$ tests. Motion responses were of greater amplitude in the occipital lobe [motion $M=$ $-.291 \mu \mathrm{V}$, nonmotion $M=0.335 \mu \mathrm{V}, t(13)=-2.50, p<$ .02 ] and parietal lobe [motion $M=0.086 \mu \mathrm{V}$, nonmotion $M=0.498 \mu \mathrm{V}, t(13)=-2.55, p<.02]$, but not in the temporal lobe [motion $M=0.0351 \mu \mathrm{V}$, nonmotion $M=$ $0.322 \mu \mathrm{V}, t(13)=-1.67, p>.05]$.

Figure $4 \mathrm{~b}$ presents scalp electrical potential distribution maps for the two periods identified as corresponding to the motion components reported by Manning et al. (1988). At $60 \mathrm{msec}$, the nonmotion response produces a negative focus centered over right-hemisphere occipital regions. The motion response is, however, characterized by a positive focus centered over right-hemisphere parietal regions. This pattern would appear to be consistent with the notion of the motion response being characterized by a widely dispersed right-hemisphere negative potential superimposed over the nonmotion response pattern. At $300 \mathrm{msec}$, the nonmotion response consists of a widely distributed positive focus centered over lefthemisphere parietal regions. The motion response provides evidence of a left-hemisphere parieto-temporal positivity, but the response is dominated by a negative focus centered over right-hemisphere occipital regions.

Distribution of motion electrical foci for horizontal and vertical AM. Scalp electrical potential distribution 


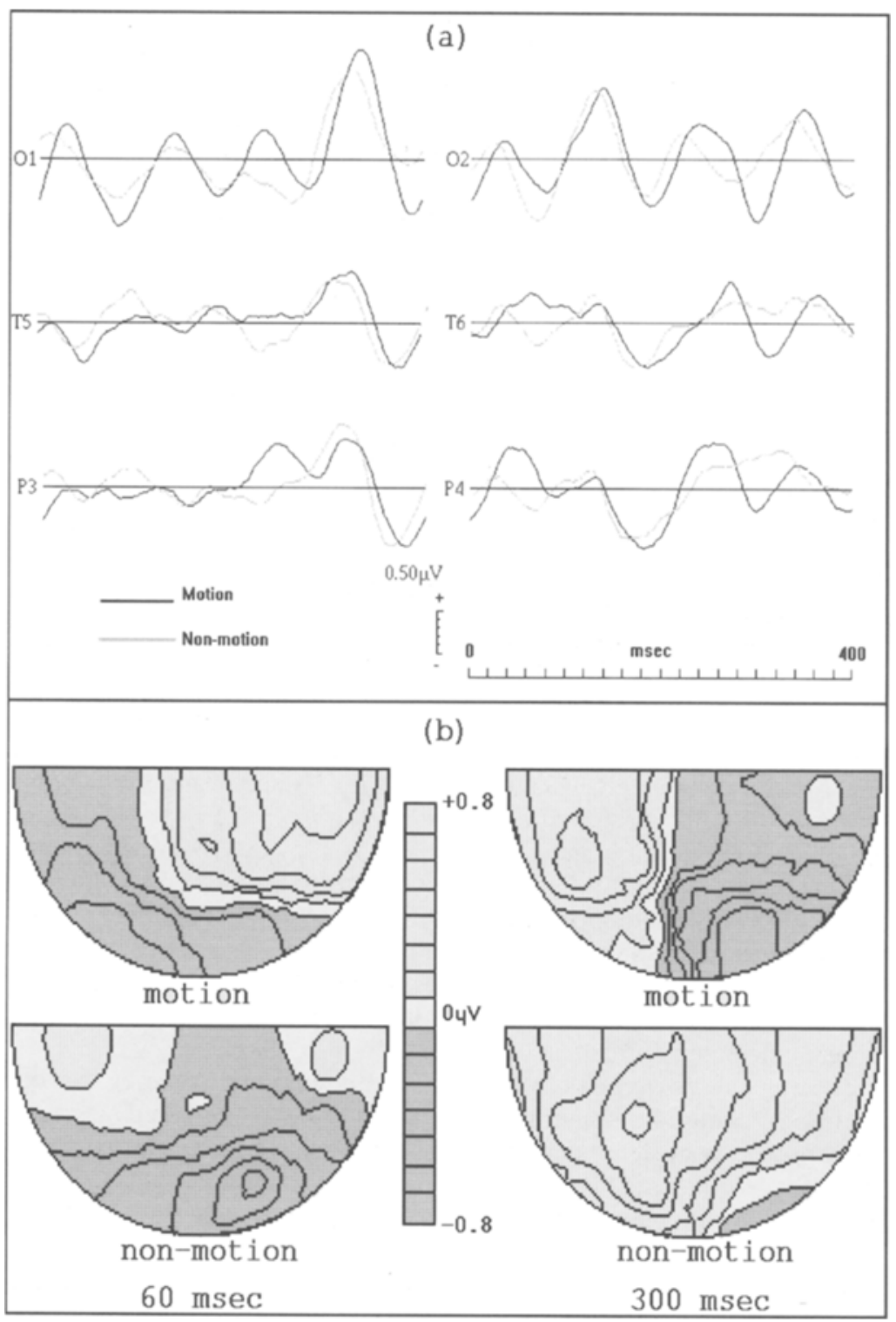

Figure 4. (a) Grand average visual evoked potentials recorded during periods in which the percept of the subject was either "motion" or "nonmotion" in response to a horizontally arranged two-element long-range apparent motion (LRAM) display. (b) Grand average scalp electrical potential distribution maps at 60 and $300 \mathrm{msec}$ for motion and nonmotion responses to a horizontally arranged two-element LRAM display.

maps were produced from the grand average VEPs for motion and nonmotion responses for both horizontal and vertical displays. Horizontal composite-flash VEPs were produced by adding left-flash and right-flash VEPs. These VEPs were used to produce composite-flash electrical potential distribution maps. A similar procedure was used to produce vertical composite-flash electrical potential distribution maps.

Maps were produced at $10-\mathrm{msec}$ intervals through the response cycle. Visual inspection of these maps demon- strated that, in general, the morphology of the motion response consisted of four negative (at approximately 0 , 100,200 , and $300 \mathrm{msec}$ ) and four positive electrical foci (at approximately 50,150,250, and $350 \mathrm{msec}$ ). Maps of these foci for motion, nonmotion, and composite flash responses are presented in Figure 5 for both horizontal and vertical displays.

Difference maps were produced by first subtracting one set of VEPs from another and then producing maps from the difference waveforms. This was done for mo- 


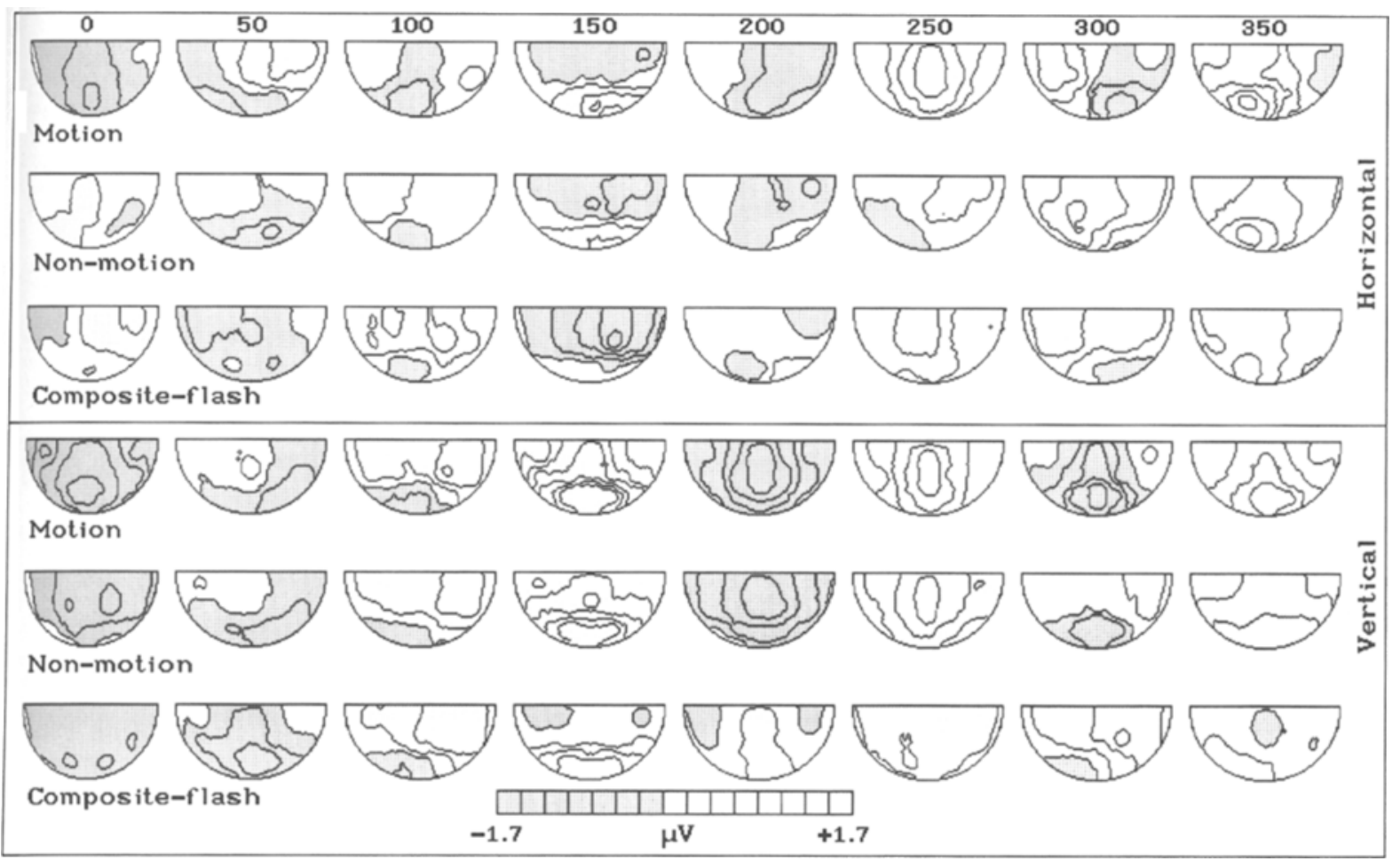

Figure 5. Grand average scalp electrical potential distribution maps at 50-msec intervals through the recording interval. Upper panel: maps in response to the horizontal display. The first row represents responses during periods in which the subjective percept is that of motion, the second represents responses during periods of nonmotion (breakdown), and the third shows synthetic responses produced by adding responses to left and right stimuli recorded in isolation. Lower panel: corresponding maps in response to vertical display.

tion minus composite flash, nonmotion minus composite flash, and motion minus nonmotion. These maps are presented in Figure 6.

Subthreshold motion responses. The maps for nonmotion minus composite flash were interpreted as representing the distribution of electrical activity associated with subthreshold activity of motion units, since these maps consisted of the activity related to form processing (composite flash) subtracted from responses that should include both form and subthreshold motion activity (nonmotion). A comparison of these maps shows that, in general, responses were quite different for vertical and horizontal displays.

For the vertical display, there was limited evidence of lateralization. At 0,300 , and $350 \mathrm{msec}$, the foci were skewed to the right hemisphere. At $50 \mathrm{msec}$, the focus was on the midline, but displayed a more rapid fall off in potential in the right hemisphere.

For the horizontal display, there was much clearer evidence of lateralization. At 0 and $250 \mathrm{msec}$, there was a negative focus in one hemisphere (right and left, respectively), and a positive focus in the other hemisphere (left and right, respectively). At 100 and $150 \mathrm{msec}$, the foci were just to the right of the midline. At 300 and $350 \mathrm{msec}$, the foci were symmetrical, but display a more rapid fall off in potential in the left hemisphere. At $50 \mathrm{msec}$, an anterior-posterior positive-negative set of foci was displayed.
Suprathreshold motion responses. The maps for motion minus nonmotion were interpreted as representing the distribution of electrical activity associated with suprathreshold activity of motion units, since these maps consisted of the activity related to form and subthreshold motion processing (nonmotion) subtracted from responses that should include form, subthreshold motion activity, and suprathreshold motion activity (motion). A comparison of maps for motion minus nonmotion demonstrates different patterns for vertical and horizontal responses. In general, the responses to vertical stimuli resulted in symmetrical patterns, with lateralized responses to the horizontal display.

For the vertical display, generally when there was a large focus, it was symmetrical. There was, however, a tendency for a more rapid fall off at the extremes in the right hemisphere $(0,150$, and $350 \mathrm{msec})$.

For the horizontal display, the foci were generally lateralized. At 50, 100, and $200 \mathrm{msec}$, there was a positive focus in one hemisphere (right, right, and left, respectively) and a negative focus in the other hemisphere. At 0 and $250 \mathrm{msec}$, the foci were symmetrical, with more rapid fall off in the right hemisphere. At $300 \mathrm{msec}$, the focus was symmetrical, with more rapid fall off in the left hemisphere. At $350 \mathrm{msec}$, there was a left-hemisphere focus.

Comparison of subthreshold and suprathreshold motion responses. For the vertical display, the subthreshold 


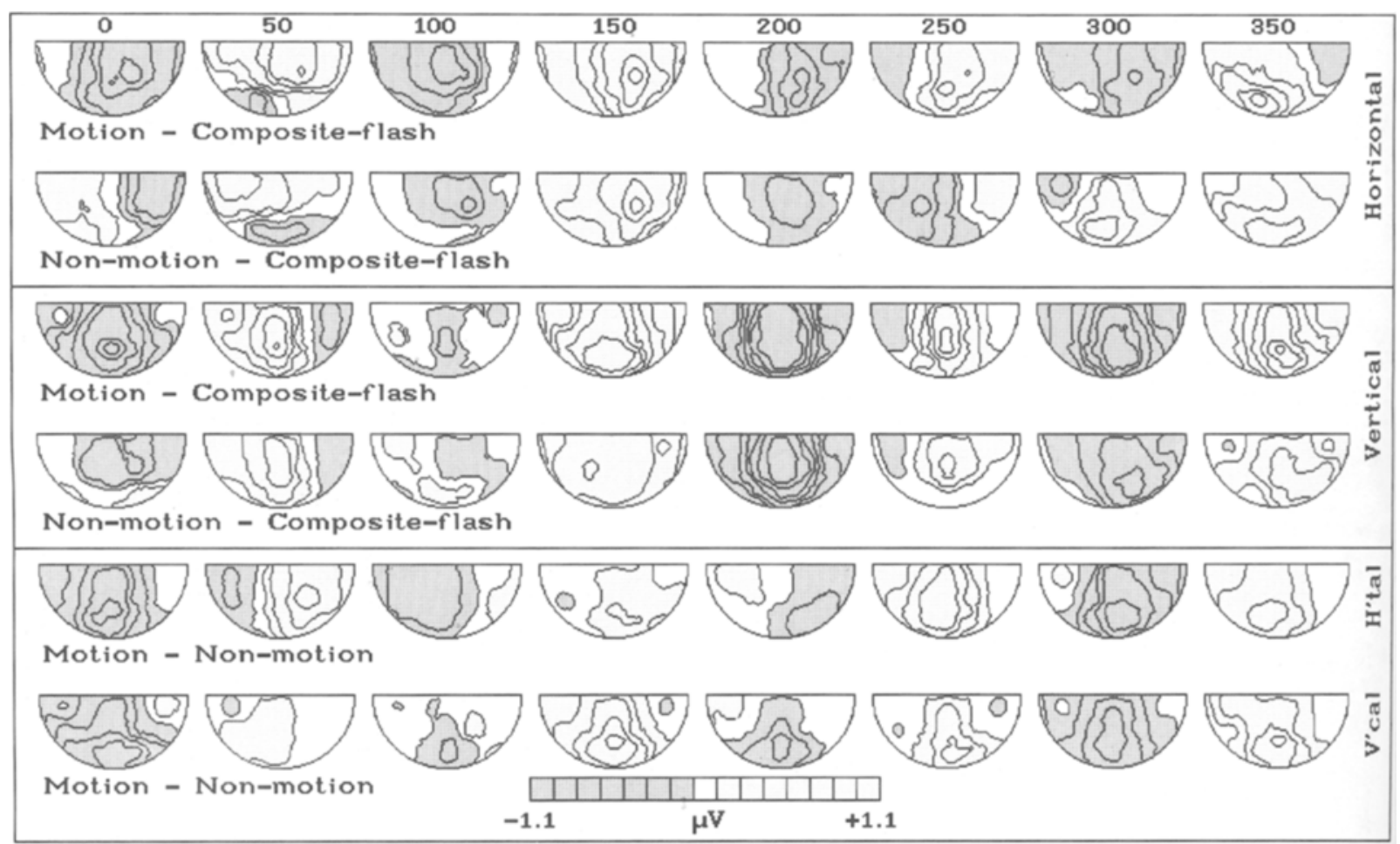

Figure 6. Grand average scalp electrical potential distribution maps at 50-msec intervals through the recording interval. Rows 1 and 2 are in response to the horizontal display and represent the distribution of the difference in electrical potential between the motion and compositeflash visual evoked potentials (VEPs) (row 1) and the nonmotion and composite-flash VEPs (row 2). Rows 3 and 4 are the corresponding maps for the vertical display. Row 5 represents the distribution of the difference in electrical potential between the motion and nonmotion VEPs for the horizontal display. Row 6 represents the distribution of the difference in electrical potential between the motion and nonmotion VEPs for the vertical display.

and suprathreshold responses were dissimilar (with the possible exception of $300 \mathrm{msec}$ ). For suprathreshold, the general pattern was a midline posterior focus, whereas for the subthreshold, the responses were generally either midline and more anterior $(50,200$, and $250 \mathrm{msec})$ than were the suprathreshold responses or were skewed to the right $(0,300$, and $350 \mathrm{msec})$.

For the horizontal display, there was also little evidence of similar distribution for subthreshold and suprathreshold motion responses. At $0 \mathrm{msec}$, the subthreshold response was lateralized, whereas the suprathreshold response was focused near the midline. At $50 \mathrm{msec}$, the subthreshold response consisted of an anterior-posterior pair of foci, whereas the suprathreshold response consisted of a left-right pair of foci. At $100 \mathrm{msec}$, the subthreshold response displayed a right-hemisphere positive focus, whereas the suprathreshold response displayed a left-hemisphere negative focus. At both 250 and $300 \mathrm{msec}$, subthreshold and suprathreshold responses displayed foci of the opposite polarity.

\section{DISCUSSION}

The present experiments offer an examination of the AM breakdown effect-a phenomenon proposed elsewhere to be the result of central adaptation processes, using TTB in response to LRAM across a range of temporal frequencies. The breakdown effect was then used in conjunction with electrophysiological techniques to establish whether the lateralized electrical responses found by Manning et al. (1988) in response to an LRAM display were the result of a lateralization of cortical motion processing or a product of the position in the visual field of their stimuli. Using the breakdown effect, the present study also aimed to establish whether suprathreshold motion responses are consistent with the notion of an increase in activity of those same motion units responding when the response is subthreshold (during periods of breakdown), or whether they are more consistent with a different population of motion units being activated.

Experiment 1 showed that for the stimuli used here, LRAM was most resistant to breakdown at $2.7 \mathrm{~Hz}$ with a falling off of mean TTB at frequencies less than $2 \mathrm{~Hz}$ and greater than $3.1 \mathrm{~Hz}$, thus providing support for Finlay and von Grünau's (1987) finding of a breakdownresistant temporal envelope within this range. Finlay and von Grünau found that TTB in LRAM was primarily related to temporal frequency, but there may be some concern in relation to the different length ISIs for each frequency and their effect on breakdown times. For example, Emerson and Gerstein (1977) found that the greatest 
neural response occurred to image movement involving short ISIs (in fact, simultaneous stimuli evoked maximal response) and small spatial displacements. In terms of an adaptation basis for the breakdown effect in AM, this evidence would provide an explanation for the short periods of motion experienced at low temporal frequencies in Experiment 1; it is more likely, however, that Emerson and Gerstein's findings relate to short-range AM rather than long-range AM. That is, it has been shown that SRAM involves a small spatial integration range of up to $20^{\prime}$ or less and prefers ISIs of no more than $40-60 \mathrm{msec}$, whereas LRAM can tolerate spatial ranges up to several degrees and operates optimally under longer ISIs than those suitable for SRAM (Braddick, 1980; see also Petersik, 1989, for a review). Moreover, Anstis et al. (1985) examined the effects of ISI using stimuli spatially separated by $2.7^{\circ}$ (i.e., LRAM stimuli) and found that at temporal frequencies ranging from 2.5 to $5.0 \mathrm{~Hz}$, AM was stronger with an ISI than without.

In order to further investigate adaptation effects, data from Experiments 2 and 3 were analyzed in relation to the proportion of motion perceived as a function of (1) time over the course of the entire experimental session and (2) time within the course of each LRAM condition block. In relation to the former, there was no evidence of LRAM perception or breakdown being influenced by block position within the entire experimental session. That is, the proportion of motion perceived did not appear to be related to whether the block was positioned during the early or late stages of the session. Given that Finlay and von Grünau's (1987) findings imply a very rapid recovery from adaptation, however, an effect over such a long period would not be expected. On the other hand, significant differences were found between temporal divisions within the LRAM block in relation to ratios of motion:nonmotion trials. The pattern of results over the course of the block for horizontal and vertical LRAM was significantly correlated, and it can be seen from Figure 3 that apart from the first temporal division, a systematic reduction in the proportion of perceived motion is evident as time progresses. Anstis et al. (1985) reported similar results using a nulling technique, wherein a reduction in the temporal frequency of the AM stimuli was used to cancel an increase in the level of adaptation. It was found that adaptation increased as a function of time elapsed, with an initial rapid increase in the amount of adaptation experienced followed by a period of slower but still increasing adaptation.

Finlay and von Grünau (1987) discussed the possibility that AM breakdown is the result of a criterion change on the part of the observer, but their data implicated exposure time as being responsible for breakdown, since periods of both motion and nonmotion perception decreased throughout the trial. In order to explain the results displayed in Figure 3, however, it may be necessary to utilize both these points. That is, the low proportions of motion perceived in the initial division of each condition might be due to a process of criterion setting, whereas the progressive decline in the amount of motion per- ceived seen in the following five divisions suggests a process of adaptation.

Experiments 2 and 3 also provide support for Finlay and von Grünau's (1987) proposition that AM breakdown is due to adaptation in central processes. For both horizontal and vertical displays, motion responses were of greater amplitude than were nonmotion responses, suggesting a reduction of cortical activity during periods of breakdown. There are also indications from physiological studies that the mechanism underlying such a reduction is centrally located. For example, Vautin and Berkley (1977) found that a response decrement in cortical cells following their activation by a moving stimulus appeared to be largely independent of any precortical adaptation that might have occurred. Furthermore, Maffei et al. (1973) demonstrated a cortical origin of response reduction when adaptation in the cortex showed interocular transfer following exposure to drifting gratings. The general pattern of results was for the composite-flash responses to be of smaller amplitude than both motion and nonmotion. This was particularly true for the vertical display. This pattern is consistent with the notion of motion responses overlaying responses to the onset and offset of the responses relating to the analysis of the form characteristics of the stimuli.

In Experiments 2 and 3, both suprathreshold distributions (motion minus nonmotion) and subthreshold distributions (nonmotion minus composite flash) were consistent with the notion that the lateralized motion responses reported by Manning et al. (1988) were the result of the position of the stimuli, rather than of a lateralization of cortical LRAM processing. Horizontally arranged stimuli (similar to those used by Manning et al.) produced examples of lateralized foci for both suprathreshold and subthreshold motion responses. When the stimuli were arranged in a vertical configuration, however, the suprathreshold and subthreshold motion foci almost fell exclusively along the midline.

Only limited evidence is available from behavioral studies of LRAM of a lateralization of AM processes. Eals (1987) proposed evidence of a right-hemisphere cortical dominance for LRAM processing. In that study, subjects were asked to predict the continuation of an AM trajectory to stimuli presented in the right and left hemifields. The measure taken on an individual trial was whether or not the correct trajectory was predicted. No measure was taken as to whether a subject reported, or experienced, the sensation of motion, and it is not clear whether the occurrence of AM was necessary for successful performance on a trial. Using stimuli similar to those used in this study, Manning (1990) presented data from 208 subjects of TTB measures across a range of temporal frequencies from a LRAM display to stimuli presented in the left and right hemifields. This study, however, yielded no evidence of hemispheric differences at any temporal frequency.

For both vertical and horizontal displays, suprathreshold and subthreshold motion responses display different scalp distributions. These data are not consistent with 
the notion that periods of motion and breakdown (nonmotion) are characterized by one population of motion units increasing and decreasing in activity. The data are more consistent with the notion that the subthreshold responses and the suprathreshold responses are the result of different populations of motion units.

Some evidence suggests that different populations of motion units are stimulated by different types of AM displays. In an investigation of VEP activity during shortrange AM, Manning and Mazzucchelli (1992) used a random-dot kinematogram (RDK) display together with a short-range two-element display that consisted of two elements from the RDK. Although the long-range-shortrange dichotomy would predict a response over occipital regions in both conditions, it was found that only the RDK display evoked such a response, whereas the twoelement display produced activity over temporal regions. Manning and Mazzucchelli argued that since occipital activity in response to the RDK did not represent the summing of activity evoked by element pairs within the display, this activity may be a reflection of a global motion process within the occipital lobe, as proposed by Singer and colleagues (see Engel, König, Kreiter, Schillen, and Singer, 1992, for a review), whereby cortical representation is based on synchronization of spatially separate groups of oscillating neurons. The responses over temporal regions were argued to be the result of motion units in areas analogous to MT of the monkey.

The use of subtraction techniques to provide "difference" VEPs to produce scalp electrical potential distribution maps to represent the distribution of electrical activity of subthreshold and suprathreshold motion responses relies on assumptions of linearity in the system. With respect to the suprathreshold responses, these assumptions would appear to be reasonable. Earlier studies (Andreassi et al., 1973; Coffin, 1977) that varied motion sensations via changes in stimulus parameters faced the potential problem of disentangling effects resulting from the motion sensation and effects resulting from changes in the stimulus array. In the present study, however, the maps representing suprathreshold responses were the result of the subtraction of responses during periods of motion and periods of breakdown (nonmotion). Both of these sets of responses were recorded in response to identical stimulus displays. Differences between motion and nonmotion conditions (and consequently the maps for motion minus nonmotion) were, therefore, in no way confounded by any change in the parameters of the stimuli.

The maps representing subthreshold motion responses are, however, more problematic. The assumption underlying the production of these maps is that the electrical responses related to the processing of form information from a two-element AM display can be approximated by the linear addition of the VEPs recorded in response to each of the elements of the display recorded in isolation. There is some evidence to support this notion. First, the data presented by Schwartz and Shagass (1964) show that at the ISIs used in this experiment, the recovery times for amplitude and latency should be sufficient for the composite-flash potentials to be used as a representation of the form response to our LRAM stimuli. Second, at least within the limits of what may be said post hoc about the results, it might be said that the data collected here at least show no inconsistency with the notion of linearity. The general pattern was that the compositeflash stimuli produced the lowest amplitude responses, the nonmotion produced the next greatest, and the motion responses produced responses of the greatest amplitude. This pattern is at least consistent with the notion of subthreshold and suprathreshold responses overlaying form responses of the type represented by our compositeflash potentials.

Statistical analyses of electrophysiological data were confined to testing whether our results are consistent with those of Manning et al. (1988). As such, responses from only two periods in the VEPs from six recording sites for the horizontal display were analyzed. The comparison showed statistically reliable differences for motion and nonmotion responses. Complementary analyses of the responses for the vertical display were not conducted as there was no a priori expectation as to which periods within the VEP would be appropriate for comparison. Statistical comparisons might have been made for each recording site for each pair of maps contrasted by visual comparison. Given the generally equivocal nature of the pattern of results regarding the principal research questions of this study, however, it is doubtful that such an approach would help to clarify the results. Further, given the extremely large number of possible comparisons, any accompanying adjustment of alpha levels would make interpretation difficult, and attempts to limit the number of comparisons might be argued to involve quite arbitrary selections.

A potential problem confronting the use of VEPs when averaging procedures are used is the intersubject variability in gross cortical anatomy. However, although Baseler, Sutter, Klein, and Carney (1994) were critical of the large stimuli used in many VEP studies, they stated that owing to their size, such stimuli can produce waveforms that are consistent across subjects. Therefore, although the stimuli used in the present study cannot be considered ideal under all circumstances, they do permit averaging of individual data. Further, the general consistency in the statistical comparison of the present horizontal VEPs with those collected by Manning et al. (1988), as well as the similarity of the present scalp electrical potential distribution maps in response to AM stimuli to those presented by Manning and Finlay (1989) and Manning and Mazzucchelli (1992), provides evidence that similar average responses were found for the groups participating across these studies.

In summary, this study collected psychophysical and electrophysiological data in an examination of the AM breakdown effect in relation to two-element LRAM displays. The data were consistent with the notion that the AM breakdown effect is the result of central adaptation processes. VEP recordings provided evidence that the 
lateralized electrical responses found by Manning et al. (1988) in response to an LRAM display were not the result of a lateralization of cortical motion processing, but rather a product of the position in the visual field of their stimuli. A comparison of subthreshold and suprathreshold VEP motion responses suggests that a different population of motion units is activated during periods of motion and breakdown in response to two-element LRAM displays.

\section{REFERENCES}

Andreassi, J. L., Mayzner, M. S., Stern, M., \& Okamura, H. (1973). Visual cortical potentials under conditions of apparent motion. Physiological Psychology, 1, 118-120.

Anstis, S. M., Giaschi, D., \& Cogan, M. (1985). Adaptation to apparent motion. Vision Research, 25, 1051-1062.

BARLOW, H. B., \& HiLL, R. M. (1963). The mechanism of directionally selective units in rabbit's retina. Journal of Physiology, 178, 447504.

Baseler, H. A., Sutter, E. E., Klein, S. A., \& Carney, T. (1994). The topography of visual evoked response properties across the visual field. Electroencephalography \& Clinical Neurophysiology, 90, 65 81 .

Braddick, O. (1974). A short-range process in apparent motion. Vision Research, 14, 519-527.

BRADDICK, O. (1980). Low-level and high-level processes in apparent motion. Philosophical Transactions of the Royal Society of London: Series B, 290, 137-151.

BURR, D., \& Ross, J. (1986). Visual processing of motion. Trends in Neurosciences, 9, 304-307.

Burr, D. C., Ross, J., \& Morrone, M. C. (1986). Smooth and sampled motion. Vision Research, 26, 643-652.

Clatworthy, J. L., \& Frisby, J. P. (1973). Real and apparent movement: Evidence for a unitary mechanism. Perception, 2, 161-164.

CofFIN, S. (1977). Cortical EEG frequency composition and the quality of apparent motion in man. Psychophysiology, 4, 586-589.

Dixon, W. J. (1985). BMDP statistical software. Berkeley: University of California Press.

EALs, M. (1987). Asymmetric processing in perception of apparent motion. Neuropsychologia, 25, 429-434.

Emerson, R. C., \& Gerstein, G. L. (1977). Simple striate neurons in the cat: II. Mechanisms underlying directional asymmetry and directional selectivity. Journal of Neurophysiology, 40, 136-155.

Engel, A. K., König, P., Kreiter, A. K., Schillen, T. B., \& Singer, W. (1992). Temporal coding in the visual cortex: New vistas on integration in the nervous system. Trends in Neurosciences, 15, 218-226.

Finlay, D. C., Manning, M. L., Neill, R. A., \& Fenelon, B. (1987).
Effects of movement in the background field on long-range apparent motion. Vision Research, 27, 1679-1682.

FINLAY, D. [C.], \& voN GrÜNAU, M. (1987). Some experiments on the breakdown effect in apparent motion. Perception \& Psychophysics, 42, 526-534.

FrISBY, J. P. (1972). Real and apparent motion. Vision Research, 12, 1051-1055.

Kolers, P. A. (1964). Illusion of movement. Scientific American, 211 , 98-108.

MAFFeI, L., Fiorentini, A., \& Bisti, S. (1973). Neural correlate of perceptual adaptation to gratings. Science, 182, 1036-1038.

MANNing, M. L. (1990). Behavioural and electrophysiological investigation of visual apparent movement. Unpublished doctoral dissertation, University of Newcastle.

ManNing, M. L., \& Finlay, D. C. (1989). Visual evoked potentials to stimuli in apparent motion. In N. W. Bond \& D. A. T. Siddle (Eds.), Psychobiology: Issues and applications (pp. 261-268). Amsterdam: Elsevier, North-Holland.

Manning, M. L., Finlay, D. C., \& Fenelon, B. (1988). Visual evoked potentials to stimuli in apparent motion. Vision Research, 28, 965-974.

Manning, M. L., \& MazzuCChELli, T. (1992). Electrical responses to short-range kinematogram displays: An occipital lobe global motion process in humans? Vision Research, 32, 447-451.

MiKaMI, A. (1991). Direction selective neurons respond to short-range and long-range apparent motion stimuli in macaque visual area MT International Journal of Neuroscience, 61, 101-112.

PANTLE, A. (1974). Motion aftereffect magnitude as a measure of the spatio-temporal response properties of direction-sensitive analyzers. Vision Research, 14, 1229-1236.

Petersik, J. T. (1989). The two-process distinction in apparent motion. Psychological Bulletin, 106, 107-127.

SchWarTz, M., \& ShaGass, C. (1964). Recovery functions of human somatosensory and visual evoked potentials. In R. Katzman (Ed.), Sensory evoked response in man (Annals of the New York Academy of Sciences, Vol. 112, pp. 510-525). New York: New York Academy of Sciences.

Steinman, S. B., \& Levi, D. M. (1992). Topography of the evoked potential to spatial localization cues. Visual Neuroscience, 8, 281-294.

VAutin, E. G., \& BerkLey, M. A. (1977). Responses of single cells in cat visual cortex to prolonged stimulus movement: Neural correlates of visual aftereffects. Journal of Neurophysiology, 40, 1051-1065. voN GRÜNAU, M. W. (1986). The motion aftereffect for long-range stroboscopic apparent motion. Perception \& Psychophysics, 40, 31-38. Wurtz, R. H., Yamasaki, D. S., Duffy, C. J., \& Roy, J.-P. (1990). Functional specialization for visual motion processing in primate cerebral cortex. Cold Spring Harbour Symposia on Quantitative Biology, 55, 717-727.

(Manuscript received July 19, 1994; revision accepted for publication May 20, 1996.) 